\title{
ALMA: $\mathrm{HI}$ and $\mathrm{He}^{+}$Lines and Dust in Starbursts, AGN and High-z Galaxies
}

\author{
Nick Scoville \\ Astronomy 249-17 \\ California Institute of Technology \\ Pasadena, CA 91125, USA \\ email: nzs@astro.caltech.edu
}

\begin{abstract}
We describe preliminary results for two ALMA projects - 1) imaging the HCN(4-3) line and H26 $\alpha$ lines in Arp 220 and 2) measurements of the dust continuum in a sample of 107 high redshift galaxies to probe the evolution of the ISM masses. The HCN observations in Arp 220 at $1 / 2^{\prime \prime}$ resolution provide the first high resolution imaging of the dense star forming gas in this prototypical ULIRG. The HCN is seen in two clearly delineated, counter-rotating disks. The H26 $\alpha$ line is a definitive probe of the star formation rate in Arp 220, avoiding obscuration by dust and contamination by AGN luminosity contributions. In the second project, the remarkable continuum sensitivity of ALMA in Band 7 is used to measure the long wavelength RayleighJeans tail of the dust emission from a sample of 120 galaxies in COSMOS at $\mathrm{z}=0.3$ to 2.2, providing estimates for the dust masses and hence their ISM masses. This technique will enable measurements for hundreds of galaxies at high-z with observations of typically $\sim 10$ min per galaxy. This is in contrast to $\mathrm{CO}$ line imaging which typically requires a few hours per galaxy even with the sensitivity of ALMA. The dust-based mass estimates also avoid the uncertainties associated with the CO-to-H2 conversion factor.
\end{abstract}

Keywords. ULIRGs; ISM: dust, gas, evolution

\section{Introduction}

ALMA provides spectacular increases in sensitivity, versatility and image fidelity over existing $\mathrm{mm} / \mathrm{submm}$ facilities. Here we briefly describe two projects conducted in Cycle0 which highlight future science which will be possible with ALMA.

\section{High Resolution Imaging in Band $7(345 \mathrm{GHz})$ of Arp 220}

Our Cycle0 project included Band 7 imaging of Arp 220 to include HCN(4-3), CS(76 ) and H26 $\alpha$. Fig. 1 shows the $349 \mathrm{GHz}$ continuum (mostly dust emission) imaged at $0.5^{\prime \prime}$ resolution, clearly showing the two nuclei separated by $1^{\prime \prime}$, corresponding to $300 \mathrm{pc}$. The peak brightness temperature in the continuum is approximately 10K. Allowing for partial covering in the source, this high brightness temperature suggests $\tau_{\text {dust }} \sim 1$, even at this long wavelength since the dust temperatures are likely in the range $50-200 \mathrm{~K}$ (based on earlier work).

The HCN(4-3) integrated emission and mean velocity are shown in Figure 2. The western nucleus shows approximately twice as much HCN emission as the east, similar to the relative fluxes seen in CO. This HCN line has a critical density $\sim 10^{6} \mathrm{~cm}^{-3}$ for collisional excitation. The velocities clearly show the counter-rotating nuclear disks in each nucleus as first detected by Sakamoto et al. (1999).

Figure 1-Right shows the integrated spectra with H26 $\alpha$ emission in Arp 220. The H26 $\alpha$ line is detected only on the western nucleus. The low n HI recombination lines provide a 

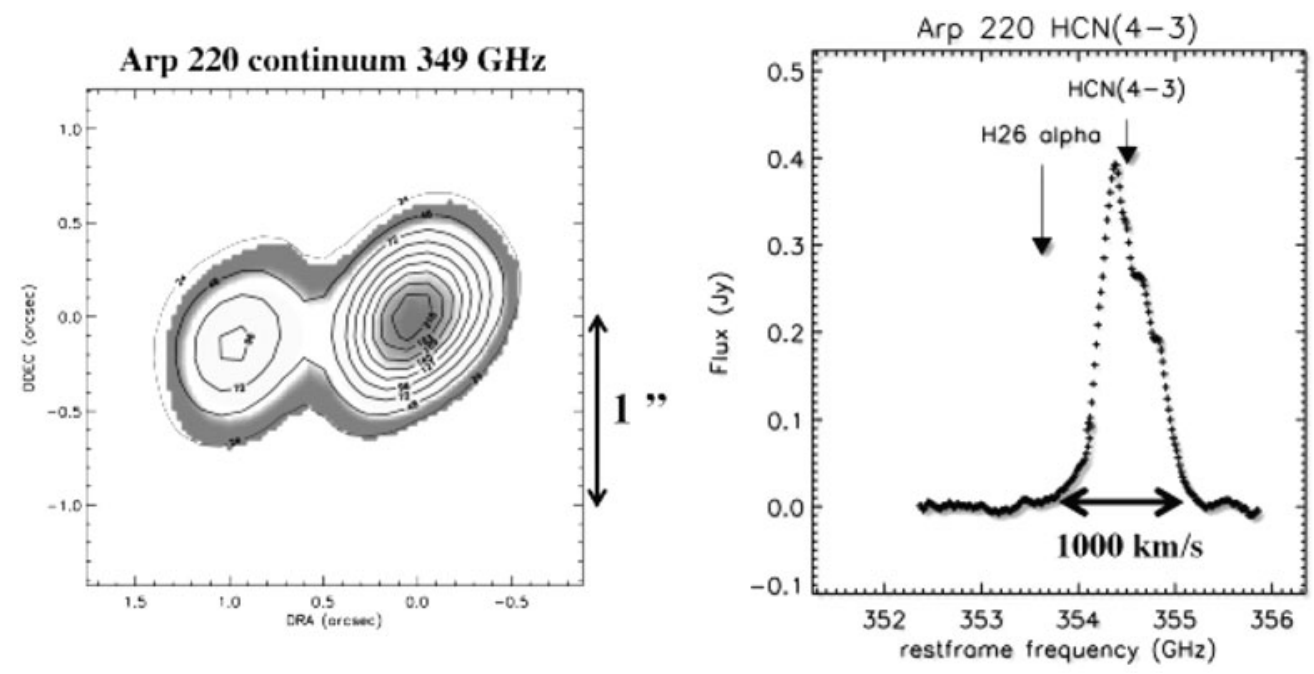

Figure 1. Left - The $349 \mathrm{GHz}$ continuum image of Arp 220 at $0.5^{\prime \prime}$ resolution. Right - Band 7 integrated spectrum covering 352 to $356 \mathrm{GHz}$ including both nuclei of Arp 220 with the continuum subtracted out. Strong HCN(4-3) emission is seen and the much weaker feature on the low frequency line wing is identified as $\mathrm{H} 26 \alpha$.
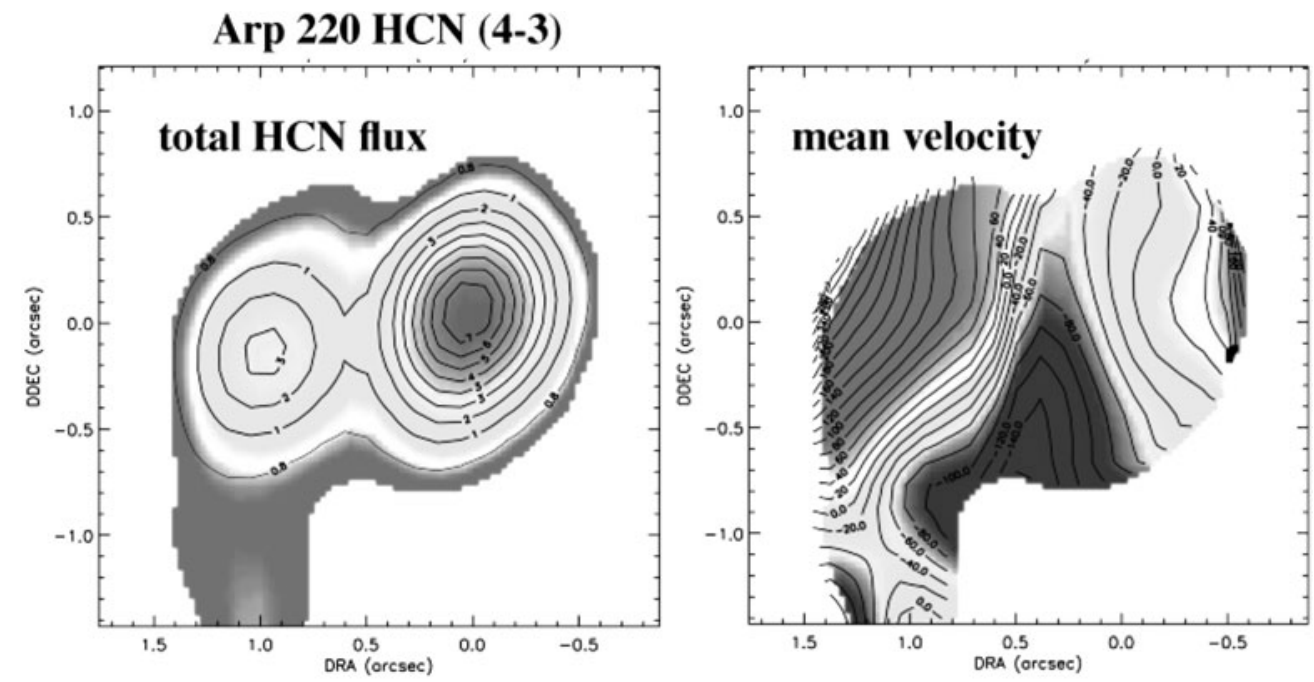

Figure 2. The total velocity-integrated $\mathrm{HCN}(4-3)$ emission and the velocity centroid are shown for Arp 220.

proportional measure of the total ionized gas emission measure $\left(E M=n_{e}^{2}\right.$ volume $)$ and hence the Lyman continuum emission rate of young OB stars. Detecting this line was a major goal of our ALMA observations, since it provides a measure of the star formation rates at a wavelength where dust obscuration is likely to be minimal. The observed flux implies a $\mathrm{SFR} \sim 150 M_{\odot} \mathrm{yr}^{-1}$ in the western nucleus.

\section{Submm Recombination Lines of $\mathbf{H} \& \mathbf{H e}^{+}$}

Our recent work on the submm recombination lines of $\mathrm{H} \& \mathrm{He}^{+}$has clearly shown that these lines will provide an accurate quantitative probe of the Lyman continuum at $>1$ 

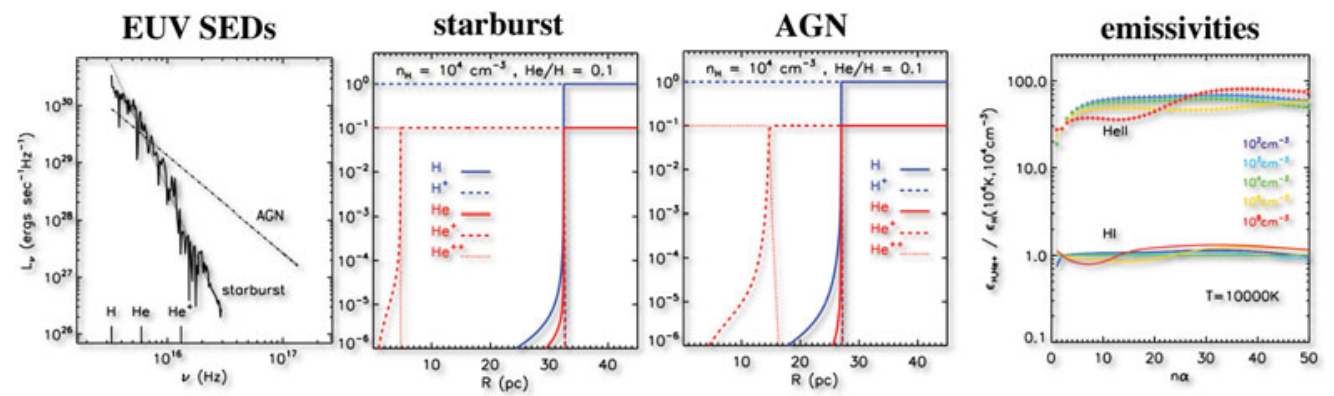

Figure 3. Left: The model EUV spectral energy distributions (SEDs) of a starburst and AGN used to calculate the ionization equilibrium structures shown in the Middle Panels for a starburst and a power-law AGN. In both cases, the total $L_{E U V}=10^{12} L_{\odot}$; it is also assumed the ionized regions are ionization-bounded and no EUV photons are absorbed by dust within the ionized regions. For the AGN source, having a harder EUV SED, the $\mathrm{He}^{++}$region is much larger - thus guaranteeing a much greater emissivity in the $\mathrm{He}^{+}$recombination lines. Right: The computed $\mathrm{HI}$ and HeII emissivities (emission rate per unit $\mathrm{n}_{e} \mathrm{n}_{\text {ion }}$ ) are shown normalized to $\mathrm{HI}$ at $10^{4} \mathrm{~K}$ for $\mathrm{n}_{e}=10^{2}$ to $10^{8} \mathrm{~cm}^{-3}$, illustrating that the emissivities for the low quantum number lines of both species are largely independent of density (and $\mathrm{T}_{e}$, see Scoville \& Murchikova 2013) and therefore reliably measure just the Lyman continuum production rates.

Rydberg (13.6 eV, the HI ionization threshold) and at > 4 Rydbergs (the $\mathrm{He}^{+}$ionization threshold) (Scoville \& Murchikova 2013). In the following subsections, we summarize the relevant results including the ionization equilibrium structure for star forming and AGN sources; the derived independence of the line fluxes on the physical conditions (density $n_{e}$ and temperature $\mathrm{T}_{e}$ ); and absence of maser amplification which hampers cm-wave recombination lines.

Approximating the EUV continuum of starbursts (using Starburst99) and AGN (power law, $L_{\nu} \propto \nu^{-1.7}$ ) (see Fig. 3 - Left), we evaluated ionization equilibrium (Fig. 3 - Middle Panels). For this case, the HeII recombination lines will be 10 - 100 times brighter relative to $\mathrm{HI}$ for the AGN case, but since the EUV hardness is very unknown for both SF and AGN, this is only illustrative.

Our analysis also indicates that the submm lines fluxes provide reliable estimates of the HII and HeIII region emission measures, hence the luminosities in the HI and HeII ionizing continua at $>13.6$ and $54.4 \mathrm{eV}$. Over the density range $n_{e}=10^{2}-10^{8} \mathrm{~cm}^{-3}$, the emissivities of the $\alpha$ lines of HI and HeII vary by less than 10\%(Fig 3-Right panel). (Variations with $T_{e}$ are also small, see Scoville \& Murchikova 2013). Importantly, we also determined that the submm lines of both species have positive opacity coefficients and hence will NOT have maser amplification.

\section{Long Wavelength Dust Continuum as a Mass Tracer}

Even with ALMA, imaging of the CO lines at $\mathrm{z} \gtrsim 1$ will typically take $>3$ hours per object having $\sim 5 \times 10^{9} M_{\odot}$ of ISM, and the samples with CO detections will therefore remain small for the near future. Here, we propose an alternate approach measuring the long-wavelength (RJ) optically thin dust continuum emission to provide a much faster (and an entirely independent) estimate for the ISM masses (HI \& $\mathrm{H}_{2}$ ) in large samples of galaxies (e.g. Eales 2012; Scoville 2012, and references therein). This technique is enabled by ALMA - Bands 6 and 7 have spectacular continuum sensitivities along with the angular resolution needed to avoid source confusion. Major advantages of this technique are: it is much faster $(<5$ min per object) than CO spectroscopy; it 
also does not require high accuracy spectroscopic redshifts; - both factors enable this approach to be extended to the very large samples of galaxies with good photometric redshifts.

At long wavelengths, the dust emission is optically thin and the observed flux density is given by

$$
S_{\nu} \propto \kappa_{d}(\nu) T_{\mathrm{d}} \nu^{2} \frac{M_{\mathrm{d}}}{d_{L}^{2}}
$$

where $T_{\mathrm{d}}$ is the temperature of the grains, $\kappa_{d}(\nu)$ is the dust opacity per unit mass of dust, $M_{\mathrm{d}}$ is the total mass of dust and $d_{L}$ is the source luminosity distance (for more details see Scoville 2014). (For simplicity in illustrating the principles, Eq. 4.1 does not include the effects of bandshifting and the compression of the frequency space which occur for high z.) In local star-forming galaxies, the majority mass of ISM dust is at $T_{d} \sim 20 \rightarrow 30 \mathrm{~K}$ (see refs in Scoville 2014) and variations in the mass-weighted dust temperatures between galaxies are small $(\sim 20-40 \mathrm{~K})$; the observed fluxes then probe the total mass of dust.

To obviate the need to know both the dust opacity and dust-to-gas ratio (which are degenerate when using Eq. 4.1 to estimate ISM masses), we have chosen instead to empirically calibrate the ratio of the specific luminosity at rest frame $850 \mu \mathrm{m}$ to total ISM mass using samples of observed galaxies, thus absorbing the opacity curve and the abundance ratio into a single empirical constant $\alpha_{850 \mu \mathrm{m}}=L_{\nu_{850 \mu \mathrm{m}}} / M_{\mathrm{ISM}}$. In Scoville (2014), three samples were developed: 1) 12 local star forming and starbursting galaxies with global SCUBA and ISM measures; 2) extensive Galactic observations from the Planck Collaboration and 3) a sample of 28 SMGs at $\mathrm{z}<3$ having $\mathrm{CO}(1-0)$ measurements. The 3 samples yielded $\alpha_{850 \mu \mathrm{m}}=1.0,0.79$ and $1.01 \times 10^{20} \mathrm{ergs} \mathrm{sec}^{-1} \mathrm{~Hz}^{-1} M_{\odot}{ }^{-1}$, respectively (see Scoville 2014). The Planck measurements are particularly convincing - they are of high SNR, span a large wavelength range and they probe the diversity of Galactic ISM including both $\mathrm{HI}$ and $\mathrm{H}_{2}$ dominated clouds, exhibiting little variation in the empirical $\alpha_{250 \mu \mathrm{m}}$ (Planck Collaboration 2011a). The Planck measurements also determine very well the long wavelength dust emissivity index: $\beta=1.8 \pm 0.1$ (Planck Collaboration 2011b). In the observations proposed here, we intentionally sample high stellar mass galaxies which should have close to solar metallicity (Erb et al. 2006) in order to avoid the issue of possible variations in dust-to-gas ratios at low metallicity. We note that Draine et al. (2007) see no variation in the dust abundance down to $\sim 20 \%$ of solar metallicity and it is reassuring that the low z sample and the high z SMGs (Fig. 4-Left and Middle) yield similar $\alpha_{850 \mu \mathrm{m}}$.

For high z galaxies, the expected flux density at observed frequency $\nu_{o b s}$ is then given by

$$
\begin{aligned}
S_{\nu_{o b s}}= & 0.83 \frac{M_{\text {ISM }}}{10^{10} M_{\odot}}(1+z)^{4.8}\left(\frac{\nu_{o b s}}{\nu_{850 \mu \mathrm{m}}}\right)^{3.8} \frac{\Gamma_{R J}}{\Gamma_{0}}\left(\frac{G p c}{d_{L}}\right)^{2} \mathrm{mJy} \\
& \text { for } \lambda_{\text {rest }} \gtrsim 250 \mu \mathrm{m}
\end{aligned}
$$

where $\Gamma$ is the correction factor for the RJ approximation (see Scoville 2014). The restriction $\lambda_{\text {rest }} \gtrsim 250 \mu \mathrm{m}$ is intended to ensure that one is on the Rayleigh-Jeans tail and that the dust is likely to be optically thin. Figure 4-right shows the expected flux as a function of redshift for the ALMA Bands 3, 4, 6 and 7 . Above $\mathrm{z}=1$, the well known "negative k-correction" causes the flux per unit ISM mass to increase at higher $\mathrm{z}$ as one moves up the far infrared SED towards the peak at $\lambda \sim 100 \mu \mathrm{m}$. Figure 4-right shows that the $350 \mathrm{GHz}$ flux density levels off and decreases above $\mathrm{z}=2.5$ (for $T_{D}=25 \mathrm{~K}$ dust). 

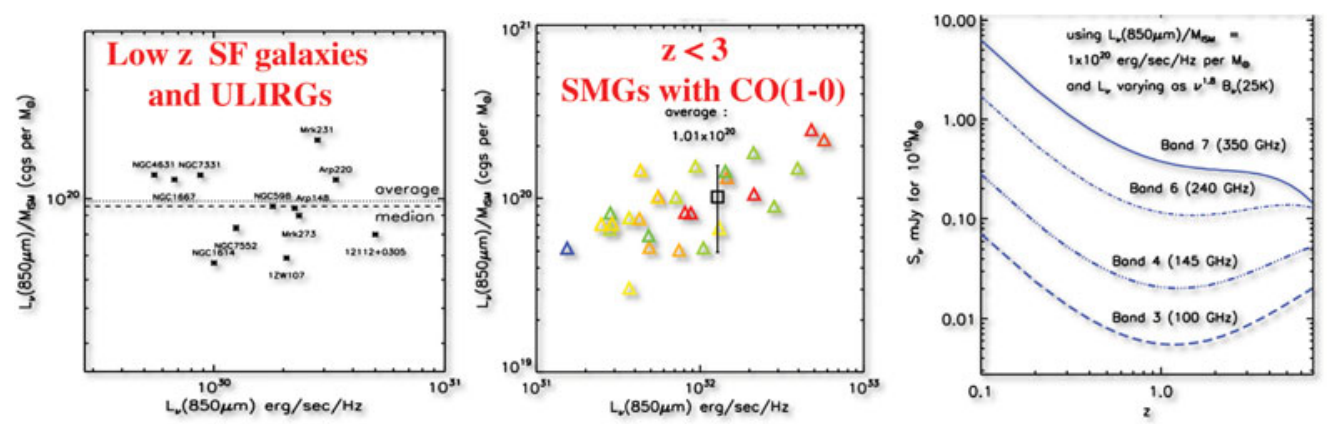

Figure 4. Left: The ratio of rest frame $850 \mu \mathrm{m}$ specific luminosity to ISM mass (determined from $\mathrm{CO}(1-0)$ and $\mathrm{HI}$ ) is shown for a sample of low-z star forming and ULIRG galaxies and (Middle) for $\mathrm{z}<3$ SMGs. The mean ratios are $\alpha_{850}=\frac{L_{\nu_{850}}}{M_{I S M}} \simeq 1 \times 10^{20} \mathrm{ergs} / \mathrm{sec} / \mathrm{Hz} / M_{\odot}$ in excellent agreement with the value obtained by Planck for ISM in the Milky Way - providing a strong empirical foundation for using the dust continuum as a quantitative probe of ISM masses. Right: The expected flux densities for the ALMA Bands are shown for a fiducial $M_{I S M}=1 \overline{\times 10^{10}} M_{\odot}$. The decreasing flux density out to $\mathrm{z} \sim 1$ is due to the increasing luminosity distances while the leveling off and rise at higher redshift is due to the "negative k-correction" as the observations probe rest frame $\lambda$ climbing up to the far infrared peak.

Above $\mathrm{z}=3$, bands 6 or 4 should be used to avoid the non-linear sensitivity to the dust temperature.

Twenty-eight of the 107 galaxies (26\%) were significantly detected. The maximum detected $350 \mathrm{GHz}$ flux was $\sim 4 \mathrm{mJy}$ and the inferred ISM masses are $1-8 \times 10^{10} M_{\odot}$. Although the detection rate is less than $50 \%$, one should note that the integration times were only 1 - 4 minutes with fewer than 18 of the eventual 64 ALMA telescopes. Secondly, this very high stellar mass sample will preferentially include a large fraction of passive galaxies and the ISM contents may be larger at lower stellar mass. The detection rate was $100 \%$ for the IR bright sample.

\section{Stacked Samples}

The mean fluxes and derived ISM masses for stacked images of each of the four galaxy subsamples may be used to explore the overall evolution of the dust emission and ISM masses. The individual galaxy images were stacked in three ways: an average, a median stack and an average weighted by the square of the rms noise. The median and weighted averages are used in the discussion and figures below. Figure 5 shows the median stacked images for each of these subsamples.

Figure 6-Left shows the average and median $350 \mathrm{GHz}$ fluxes for each of the four stacked samples. All four samples are significantly detected in the stacked images (both median and average). The lowest redshift sample does not have high statistical significance in the stacked detection but that is to be expected, given the anticipated evolution of ISM masses for these very massive galaxies. The derived ISM masses for each of the samples are shown in Figure 6-Right for the median stacks. The figure clearly show an increase in the ISM content both in absolute mass and gas mass fraction (since the stellar masses are the same) from $\mathrm{z}=0.4$ to 1 . From $\mathrm{z}=1$ to 2 , the ISM mass shows no significant evolution for the strictly stellar mass-selected samples. This is a very interesting result, but given the small sample sizes and the fact that these are only the most massive galaxies, it is not clear how general these results are in terms of overall cosmic evolution. A high priority should be placed on extending the samples to lower stellar mass and better sampling of 

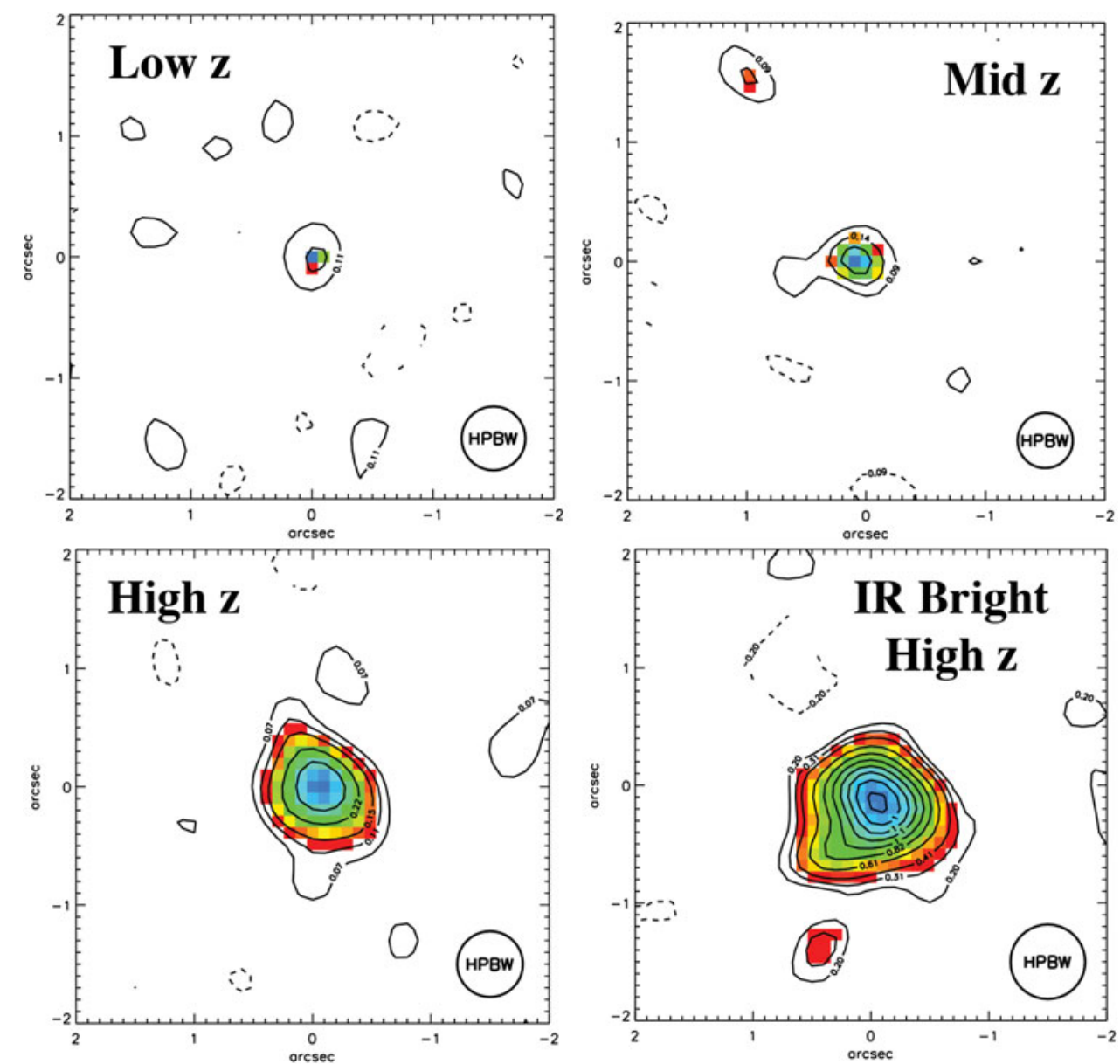

Figure 5. Median stacked images for the four subsamples of galaxies. The scale is in arcsecs and the contours are at $-2,2,3,4,5,7,9,11 \sigma$.

the star formation characteristics. Comparing the IR bright sample at $\mathrm{z}=2$ with the strictly mass-selected sample at the same redshift, it is clear that the IR bright galaxies have considerably higher long wavelength fluxes and presumably higher ISM contents by a factor $\sim 5$. This increase in the apparent ISM mass is in fact larger than the increase in the estimated SFRs. If this holds up, it would clearly suggest that the $\mathrm{z}=2 \mathrm{IR}$ bright galaxies are extra luminous simply because they have more ISM.

The depletion timescales for the ISM via star formation are given by $\tau_{S F}=M_{I S M} /<$ $S F R>$. This depletion timescale is similar for the subsamples (0.2 - $1.2 \mathrm{Gyr})$. Both of the $\mathrm{z} \sim 2$ samples have similar depletion timescales $(0.2$ and $0.5 \mathrm{Gyr}$ for the normal and IR bright samples, respectively). This suggests that the IR bright galaxies have more ISM and are using the gas with a higher star formation efficiency.

The gas mass fractions $\left(M_{I S M} /\left(M_{*}+M_{I S M}\right)\right)$ are $\sim 2 \pm 0.5,12 \pm 3,14 \pm 3$ and $53 \pm 5 \%$ for the low-z, mid-z, high-z and IR bright samples, respectively. Tacconi (2013) surveyed 52 galaxies at $\mathrm{z}=1-3$ in $\mathrm{CO}(3-2)$ and found mean gas mass fractions of 0.33 and 0.47 at $\mathrm{z} \sim 1.2$ and 2.2 , respectively. Their sample was selected with $M_{*}>2.5 \times 10^{10} M_{\odot}$ and $\mathrm{SFR}>32 M_{\odot} \mathrm{yr}^{-1}$; it is therefore likely to favor more active star forming galaxies than our purely stellar-mass selected samples. On the other hand, our IR-bright sample is 

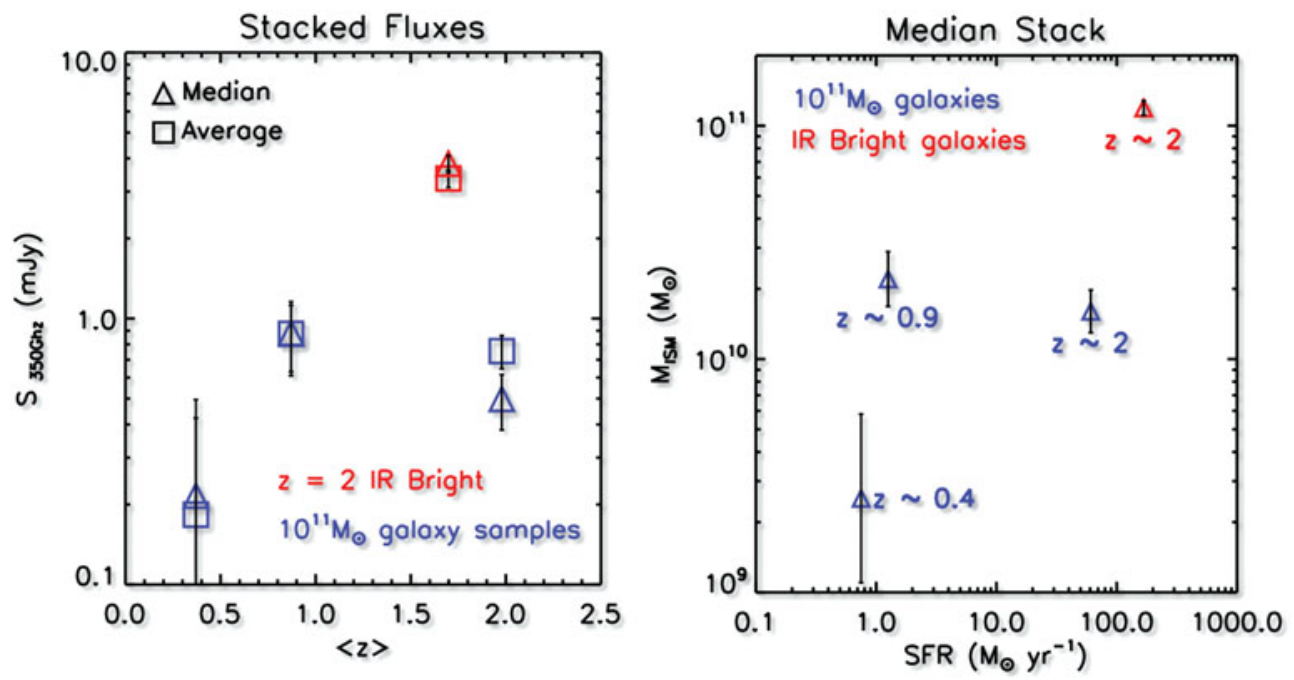

Figure 6. Left: The $350 \mathrm{GHz}$ continuum fluxes from stacked images of the four galaxies samples. Median and average stacked images are indicated by triangles and squares. The signal-to-noise ratios are from $\sim 3,4,8$ and 16 for the low-z, mid-z, high-z and IR bright samples, respectively. Right: Derived ISM masses are shown with their median SFRs. The star formation timescales $\left.\left.\overline{\left(\tau_{S F}\right.}=M_{I S M} /<S F R\right\rangle\right)$ are in the range 0.2 to $1.2 \mathrm{Gyr}$.

likely to be more star forming than their average galaxy so it is entirely reasonable to find a somewhat higher gas mass fraction.

\section{References}

Draine, B. T., et al. 2007, ApJ 663, 866

Eales, S., et al. 2012, ApJ 761, 168

Erb, D. K., Shapley, A. E., Pettini, M., Steidel, C. C., Reddy, N. A., \& Adelberger, K. L. 2006, ApJ 644, 813

Planck Collaboration 2011a, A\&SA 536, A21

Planck Collaboration 2011b, A\&A 536, A25

Sakamoto, K., Scoville, N. Z., Yun, M. S., Crosas, M., Genzel, R., \& Tacconi, L. J. 1999, ApJ 514,68

Scoville, N. \& Murchikova, L. 2013, ApJ 779, 75

Scoville, N. Z. 2012, ArXiv e-prints

Scoville, N. Z., et al. 2014, ApJ 000, 000

Tacconi, L. J., et al. 2013, ApJ 768, 74 